\title{
Borel-Le Roy Summability of the High Temperature Expansion for Classical Continuous Systems
}

\author{
Marco Maioli \\ Dipartimento di Matematica pura e applicata, Università di Modena, I-41100 Modena, Italy
}

\begin{abstract}
For classical gases with suitable pair interactions such that $\Phi(r) \sim\left(\ln r^{-1}\right)^{p}$ as $r \rightarrow 0(p \in \mathbb{N})$, the Taylor expansion in $\beta$ of the correlation functions and the pressure are summable at $\beta=0$ by the Borel-Le Roy method of order $p+1$.
\end{abstract}

\section{Introduction}

As it is known [5], for classical continuous systems with stable and regular pair potentials the correlation functions and the pressure admit a convergent power series expansion in the activity $z$, while the typical analyticity region in $\beta$ $\left(\beta=(k T)^{-1}\right)$ is the half plane $\operatorname{Re} \beta>0$. As recently proved by Wagner [7], if the pair potential is bounded and absolutely integrable, the correlation functions and the pressure turn out to have Borel summable Taylor expansions at $\beta=0$ (for Borel summability, see e.g. $[4,6])$. Among other facts the proof uses analyticity for $\operatorname{Re} \beta>0$ and the bound $\int|\Phi(x)|^{n} d x \leqq\left(\|\Phi\|_{\infty}\right)^{n-1}\|\Phi\|_{1}$.

Here the aim is to prove the Borel-Le Roy summability $([3,2])$ of these power series, under suitable hypotheses on the pair potential $\Phi(r)$. Hypotheses (1), (2), (3) below include, in particular, the asymptotic behaviour $\Phi(r) \sim\left(\ln r^{-1}\right)^{p}$ as $r \rightarrow 0$ $(p \in \mathbb{N})$. These assumptions allow us to analytically continue the correlation functions beyond the right half plane, to a region containing $\left\{\beta / \operatorname{Re} \beta^{\frac{1}{1+p}}>0\right\}$ on the Riemann surface of $\ln \beta$, which is suggested by the analytic structure of $\int\left(e^{-\beta \Phi(x)}-1\right) d x$ in these cases (Proposition 2.1). Moreover the power series remainders are proved not to grow faster than $((p+1) n)$ !, which is somehow suggested by bounds of the type $\int|\Phi(x)|^{n} d x \leqq c(p n)$ !, and by a further factor $(n !)^{2}$ that can be expected in the estimates of $n^{\text {th }}$ derivatives of correlation functions.

In the case $v=2, p=1$, conditions (1), (2), (3) include potentials exponentially decreasing as $r \rightarrow+\infty$ and with the asymptotic behaviour of two-dimensional Yukawa potentials (see e.g. $[8,1])$ as $r \rightarrow 0$, although $\Phi(r)=e^{-a r}\left(\ln r^{-1}\right)$ is not in this 
class owing to the technical requirement of the existence of an inverse function $r=\Psi(t)$ [Hypothesis (1)].

\section{Notations}

Let us assume the following hypotheses on the pair potential $\Phi(r), r>0$ :

(1) $\Phi(r)$ is the restriction to $r \in \mathbb{R}_{+}$of a function analytic in some angular sector containing $\mathbb{R}_{+}$, which admits an inverse function $\Psi(t)$ analytic for $|\arg (t)|<p \pi / 2$ (for some $p \in \mathbb{N}$ );

(2) $\Phi(r) \sim c\left(\ln r^{-1}\right)^{p}$ as $r \rightarrow 0$, for some $c>0$;

(3) $\Phi(r) \sim c^{\prime} e^{-a r} r^{n}(\ln r)^{m}$ as $r \rightarrow+\infty$, for some $c^{\prime}, a>0, n, m \in \mathbb{Z}$. As a consequence, taking from now on $c=c^{\prime}=1$, the inverse function $\Psi(t)$ admits the asymptotic behaviours:

$$
\begin{array}{ccc}
\Psi(t) \sim \exp \left(-t^{1 / p}\right) & \text { as } & t \rightarrow \infty \\
\Psi(t) \sim a^{-1} \ln t^{-1} & \text { as } & t \rightarrow 0
\end{array}
$$

in the analyticity sector.

An example is provided by $\Phi(r)=e^{-r}\left(\ln r^{-1}\right)^{p}(1-r)^{-p}$.

By these assumptions $\Phi(r)$ is a monotone and positive potential and it satisfies stability and regularity [5], with stability constant given by zero. Then, in order to represent the infinite volume correlation functions [5, Chap. 4.2], on the space $E$ of sequences $\varphi=\left(\varphi(x)_{n}\right)_{n \in \mathbb{N}}$ of complex functions such that

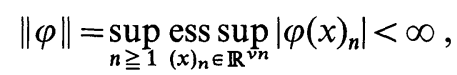

we can define the operator $\Gamma_{\beta}$ such that

$$
\begin{gathered}
\left(\Gamma_{\beta} \varphi\right)\left(x_{1}\right)=\sum_{n=1}^{\infty}(n !)^{-1} \int d(y)_{n} K_{\beta}\left(x_{1},(y)_{n}\right) \varphi(y)_{n}, \\
\left(\Gamma_{\beta} \varphi\right)(x)_{m}=\varphi(x)_{m-1}^{\prime}+\sum_{n=1}^{\infty}(n !)^{-1} \int d(y)_{n} K_{\beta}\left(x_{1},(y)_{n}\right) \varphi\left((x)_{m-1}^{\prime},(y)_{n}\right),
\end{gathered}
$$

where $(x)_{m-1}^{\prime}=\left(x_{2}, x_{3}, \ldots, x_{m}\right)$ and

$$
K_{\beta}\left(x_{1},(y)_{n}\right)=\prod_{j=1}^{n}\left(\exp \left(-\beta \Phi\left(x_{1}-y_{j}\right)\right)-1\right) .
$$

On the same space we can define

where

$$
\left(\Delta_{\beta} \varphi\right)(x)_{m}=\exp \left(-\beta W^{1}(x)_{m}\right) \varphi(x)_{m},
$$

$$
W^{1}(x)_{m}=0 \quad \text { for } \quad m=1, \quad W^{1}(x)_{m}=\sum_{j=2}^{m} \Phi\left(x_{1}-x_{j}\right) \text { for } m \geqq 2 .
$$

If $\operatorname{Re} \beta>0, \mathbb{K}_{\beta}=\Delta_{\beta} \Gamma_{\beta}$ is a product of bounded operators in $E$ and $\left\|\mathbb{K}_{\beta}\right\|$ $\leqq \exp (C(\beta))$, where $C(\beta)=\int\left|e^{-\beta \Phi(x)}-1\right| d x<\infty$ by regularity. For

$$
|z|<\exp (-C(\beta)), \quad \operatorname{Re} \beta>0,
$$


the sequence of the infinite volume correlation functions belongs to $E$ and can be written as:

$$
\varrho(\beta, z)=\left(\mathbb{I}-z \mathbb{K}_{\beta}\right)^{-1} z \alpha
$$

where $\alpha\left(x_{1}\right)=1, \alpha(x)_{m}=0$ for $m>1$.

Under assumptions (1), (2), (3) we can consider the extended function $\tilde{C}(\beta)$ defined in the following proposition.

Proposition 2.1. The integral $\int_{\mathbb{R}^{v}}\left(e^{-\beta \Phi(x)}-1\right) d x$ admits an analytic extension $\widetilde{C}(\beta)$ for $\operatorname{Re} \beta^{(1+p)^{-1}}>0$, such that $|\widetilde{C}(\beta)| \leqq k|\beta|(k>0)$ uniformly with respect to the phase of $\beta$.

Proof. By assumption (1), for $\beta>0$, the above integral is equal to

$$
\begin{gathered}
k_{1} \int_{0}^{\infty}\left(e^{-\beta t}-1\right) \Psi(t)^{v-1} \Psi^{\prime}(t) d t=k_{1} \int_{0}^{\infty}\left(\exp \left(-\beta^{(1+p)^{-1}} \tau\right)-1\right) \\
. \Psi\left(\tau \beta^{-p(1+p)^{-1}}\right)^{v-1} \Psi^{\prime}\left(\tau \beta^{-p(1+p)^{-1}}\right) \beta^{-p(1+p)^{-1}} d \tau .
\end{gathered}
$$

The last integral is absolutely convergent for $\operatorname{Re} \beta^{(1+p)^{-1}}>0$. Indeed, setting $\beta=|\beta| e^{i \theta}, \tau=\sigma|\beta|^{p(p+1)^{-1}}$ and using assumption (1):

$$
\begin{aligned}
|\tilde{C}(\beta)| \leqq & k_{1} \int_{0}^{\infty} \mid\left(1-\exp \left(-|\beta| \sigma e^{i \theta(p+1)^{-1}}\right)\right) \\
& \cdot \Psi\left(\sigma e^{-i \theta p(p+1)^{-1}}\right)^{\nu-1} \Psi^{\prime}\left(\sigma e^{-i \theta p(p+1)^{-1}}\right) \mid d \sigma .
\end{aligned}
$$

Since $e^{-z}-1=-z e^{-\varepsilon z}$ for some $\varepsilon=\varepsilon(z), 0 \leqq \varepsilon \leqq 1$, we have by (4),(5):

$$
\begin{aligned}
|\tilde{C}(\beta)| \leqq & k_{2} \int_{0}^{1}|\beta| \sigma \exp \left(-\varepsilon|\beta| \sigma \cos \frac{\theta}{p+1}\right)\left|\ln \sigma^{-1}\right|^{v-1} \sigma^{-1} d \sigma \\
& +k_{2} \int_{1}^{\infty}|\beta| \sigma \exp \left(-\varepsilon|\beta| \sigma \cos \frac{\theta}{p+1}\right) \\
& \cdot \exp \left(-v \sigma^{p^{-1}} \cos \frac{\theta}{p+1}\right) \sigma^{p^{-1}-1} d \sigma \leqq k_{3}|\beta|
\end{aligned}
$$

if $|\theta|<(p+1) \pi / 2$, for some $k_{2}, k_{3}>0$. The uniformity of (14) with respect to $\theta$ can be checked by the equivalent substitution $t=\left(\beta e^{i \gamma}\right)^{p(p+1)^{-1}}$ in (12), with $\gamma$ real and small. Indeed the consideration of complex $\beta$ leads to the estimate (14) with $\theta$ replaced by $\theta+\gamma$ : whence the uniformity near $\theta=-(p+1) \pi / 2$ and $\theta=(p+1) \pi / 2$ by assuming $\gamma>0$ and $\gamma<0$ respectively, and the assertion is proved.

Let $z \in \mathbb{C}^{v}$ : we say that $|\operatorname{Im} z| \leqq d$ if the imaginary part of each component is not larger than $d$. Let $S_{\delta}^{n}=\left\{(z)_{n} \in \mathbb{C}^{v n} /\left|\operatorname{Im} z_{j}\right| \leqq \delta\right.$ for $\left.j=1,2, \ldots, n\right\}$ and let $T_{\delta}^{n}=\left\{(z)_{n} \in S_{\delta}^{n} / z_{1}=z_{j}\right.$ for some $\left.j \neq 1\right\}$. We can consider the space $F_{\delta}$ of sequences of functions $\varphi(z)_{n}$ analytic [in each one of the $v n$ components of $(z)_{n}$ ] at least on $S_{\delta}^{n} \backslash T_{\delta}^{n}$ and bounded on $S_{\delta}^{n}$, such that

$$
\|\varphi\|_{\delta}=\sup _{n \geqq 1} \sup _{(z)_{n} \in S_{\delta}^{n}}\left|\varphi(z)_{n}\right|<\infty .
$$

Of course $\alpha \in \bigcap_{\delta>0} F_{\delta}$ and $\|\alpha\|_{\delta}=1$ for all $\delta$. Moreover, by the properties of $e^{-\beta \Phi}$ on $\mathbb{C}^{v}, \varrho(\beta, z)$ belongs to these spaces for $\beta>0$. 


\section{Analytic Continuation and Estimates}

Proposition 3.1. There is some $d>0$ such that if $\varphi \in F_{q d}(q>1)$ the expressions (6), (7) admit analytic continuation $\left(\tilde{\Gamma}_{\beta} \varphi\right)(x)_{m}$ to $\operatorname{Re} \beta^{(1+p)^{-}}>0$ such that $\left(\tilde{\Gamma}_{\beta} \varphi\right) \in F_{(q-1) d}$ and

$$
\left\|\tilde{\Gamma_{\beta}} \varphi\right\|_{(q-1) d} \leqq k_{1} \exp \left(k_{2}|\tilde{C}(\beta)|\right)\|\varphi\|_{q d}
$$

with $k_{1}, k_{2}$ independent of $q$.

Proof. It is sufficient to consider (7). For $\beta>0$ :

$$
\begin{aligned}
\left(\Gamma_{\beta} \varphi\right)(x)_{m}= & \varphi(x)_{m-1}^{\prime}+\sum_{n=1}^{\infty}(n !)^{-1} \\
& \left.\cdot \int_{(\mathbb{R}+)^{n}} \int_{T_{n}} \prod_{j=1}^{n}\left(e^{-\beta \Phi\left(r_{j}\right)}-1\right)(-1)^{n} r_{j}^{v-1} d r_{j}\right) \\
& \cdot \varphi\left((x)_{m-1}^{\prime}, x_{1}-r_{1} f_{1}, \ldots, x_{1}-r_{n} f_{n}\right) d \mu_{n} .
\end{aligned}
$$

In these integrals $r_{j}=\left|x_{1}-y_{j}\right|(j=1,2, \ldots, n), x_{1}-y_{j}=r_{j} f_{j}$, where $f_{j}$ only depends on the angular part of the $v$-dimensional polar coordinates, and $\int_{T_{n}} d \mu_{n}$ denotes the integration over such angular coordinates for all $j$. By the substitution $r_{j}=\Psi\left(\beta^{-p(1+p)^{-1}} t_{j}\right)$

$$
\begin{aligned}
\left(\Gamma_{\beta} \varphi\right)(x)_{m}= & \varphi(x)_{m-1}^{\prime}+\sum_{n=1}^{\infty}(n !)^{-1} \int_{\left(\mathbb{R}_{+}\right)^{n}} \int_{T_{n}} \prod_{j=1}^{n}\left(\left(1-\exp \left(t_{j} \beta^{(1+p)^{-1}}\right)\right)\right. \\
& \left.\cdot \Psi\left(t_{j} \beta^{-p(1+p)^{-1}}\right)^{v-1} \Psi^{\prime}\left(t_{j} \beta^{-p(1+p)^{-1}}\right) d t_{j}\right) \beta^{-p n(1+p)^{-1}} \\
& \cdot \varphi\left((x)_{m-1}^{\prime}, x_{1}-\Psi\left(t_{1} \beta^{-p(p+1)^{-1}}\right) f_{1}, \ldots, x_{1}\right. \\
& \left.-\Psi\left(t_{n} \beta^{-p(1+p)^{-1}}\right) f_{n}\right) d \mu_{n},
\end{aligned}
$$

where the $f_{j}^{\prime}$ s are independent of $\beta$ and $t_{j}$. Now, the right-hand-side of (18) makes sense as an analytic function of $\beta$ for $\operatorname{Re} \beta^{(1+p)^{-1}}>0$. Indeed the integrand is analytic by assumption (1). Moreover, after the substitution $t_{j}=\tau_{j}|\beta|^{(1+p)^{-1} p}$ we have by (4), (5):

$$
\begin{array}{ccc}
\Psi\left(\tau e^{-i \theta p(1+p)^{-1}}\right) \sim \exp \left(-\tau^{p^{-1}} e^{-i \theta(1+p)^{-1}}\right) & \text { as } & \tau \rightarrow \infty, \\
\operatorname{Im} \Psi\left(\tau e^{-i \theta p(1+p)^{-1}}\right) \sim-a^{-1} i p \theta(1+p)^{-1} & \text { as } & \tau \rightarrow 0 .
\end{array}
$$

As a consequence:

$$
\left|\operatorname{Im} \Psi\left(\tau e^{-i \theta p(1+p)^{-1}}\right)\right| \leqq d
$$

for some $d>0$, uniformly for $|\theta| \leqq(p+1) \pi / 2$. On the other hand $\left|f_{j}\right| \leqq 1$, therefore:

$$
\left|\varphi\left((x)_{m-1}^{\prime}, x_{1}-\Psi\left(\tau_{1} e^{-i \theta p(1+p)^{-1}}\right) f_{1}, \ldots\right)\right| \leqq\|\varphi\|_{q^{d}}
$$

if $\left|\operatorname{Im} x_{1}\right| \leqq(q-1) d$. Comparing (18) and (21) with (12) we obtain (16) and the assertion is proved.

Proposition 3.2. Let $\varphi \in F_{q d}$ (q, $d$ as in Proposition 3.1). For fixed $R>0$ there are $A_{1}, A_{2}$ such that, for $\operatorname{Re} \beta^{(1+p)^{-1}}>0,|\beta|<R$,

$$
\left|D_{\beta}^{s}\left(\tilde{\Gamma_{\beta}} \varphi\right)(x)_{m}\right| \leqq A_{1}\left(A_{2}\right)^{s}((p+1) s) !\|\varphi\|_{q d}
$$

for $(x)_{m} \in S_{(q-1) d}^{m}, s \in \mathbb{N}_{0}$. 
Proof. It is sufficient to consider the $m>1$ cases and to bound the $s^{\text {th }}$ derivative with respect to $|\beta|$. By the substitution $t_{j}=\tau_{j}|\beta|^{p(1+p)^{-1}}$ in (18), the only term depending on $|\beta|$ is

$$
\prod_{j=1}^{n}\left(\exp \left(-|\beta| \tau_{j} e^{i \theta(1+p)^{-1}}\right)-1\right)
$$

Hence, by the same argument of Proposition 3.1 we have:

$$
\begin{aligned}
\left|D_{|\beta|}^{s}\left(\tilde{\Gamma_{\beta}} \varphi\right)(x)_{m}\right| \leqq & \|\varphi\|_{q d}+\sum_{n=1}^{\infty}(n !)^{-1} \sum_{\begin{array}{c}
s_{1}, \ldots, s_{n} \geqq 0 \\
s_{1}+\ldots+s_{n}=s
\end{array}} \frac{s !}{s_{1} ! \ldots s_{n} !} \cdot\|\varphi\|_{q d} \\
& \cdot \prod_{j=1}^{n}\left(\int_{\mathbb{R}_{+}} \mid D_{|\beta|}^{s_{j}}\left\{\operatorname { e x p } \left(-|\beta| \tau_{j} e^{\left.\left.i \theta(1+p)^{-1}\right)-1\right\}}\right.\right.\right. \\
& \left.\cdot \Psi\left(\tau_{j} e^{-i \theta p(1+p)^{-1}}\right)^{v-1} \Psi^{\prime}\left(\tau_{j} e^{-i \theta p(1+p)^{-1}}\right) d \tau_{j} \mid\right) .
\end{aligned}
$$

Now, by (19) [compare with (14)],

$$
\begin{aligned}
\int_{\mathbb{R}_{+}}\left|D_{|\beta|}^{s}\left\{\exp \left(-|\beta| \tau e^{i \theta(1+p)^{-1}}\right)-1\right\} \Psi\left(\tau \mathrm{e}^{-i \theta p(1+p)^{-1}}\right)^{\nu-1} \Psi^{\prime}\left(\tau e^{-i \theta p(1+p)^{-1}}\right) d \tau\right| \\
\leqq k_{1} \int_{0}^{1}|\beta| \tau^{s+1}|\ln \tau|^{\nu-1} \tau^{-1} d \tau+k_{2} \int_{1}^{\infty}|\beta| \tau^{s+1} \\
\quad \exp \left(-v \tau^{p^{-1}} \cos \frac{\theta}{p+1}\right) \tau^{p^{-1}-1} d \tau \leqq k_{3}(p s) !\left(k_{4}\right)^{s}
\end{aligned}
$$

where the constants are independent of $|\beta|$ for $|\beta|<R$ and can be chosen independent of $\theta$ by the argument used in Proposition 2.1. By combining (23) and (24):

$$
\left|D_{|\beta|}^{s}\left(\tilde{\Gamma}_{\beta} \varphi\right)(x)_{m}\right| \leqq\|\varphi\|_{q d} \sum_{n=0}^{\infty}(n !)^{-1} A^{s}(s !)\left(k_{3}\right)^{n}(p s) !\left(k_{4}\right)^{s},
$$

since $s_{1}+s_{2}+\ldots+s_{n}=s$, and the estimate (22) is proved.

Proposition 3.3. There is a scale of spaces $F_{\delta, h} \subset F_{\delta, h-1} \subset \ldots \subset F_{\delta, 0}=F_{\delta}$ (with norms $\|\cdot\|_{\delta, h}, \delta>0, h \in \mathbb{N}_{0}$ ) such that, if $|\beta|<R,(9)$, (10) define a bounded operator $\Delta_{\beta}$ from $F_{\delta, h+1}$ to $F_{\delta, h}$ and

$$
\left\|\left(D_{\beta}^{s} \Delta_{\beta}\right) \varphi\right\|_{\delta, h} \leqq\left(A_{3}\right)^{s}(s !)\|\varphi\|_{\delta, h+1}
$$

uniformly for $s \in \mathbb{N}_{0}, \delta>0, h \in \mathbb{N}_{0},|\beta|<R$.

Proof. We can simply consider the space $F_{\delta, h}$ of vectors $\varphi \in F_{\delta}$ such that

$$
\|\varphi\|_{\delta, h}=\sup _{m \geqq 1} \sup _{(x)_{m} \in S_{\delta}^{m}} \exp \left(h R^{\prime}\left|W^{1}(x)_{m}\right|\right)\left|\varphi(x)_{m}\right|<\infty
$$

where $R^{\prime}>R$ and $W^{1}(x)_{m}$ is defined by (10). Then the first assertion is immediate. 
Since

$$
\begin{aligned}
& \left(D_{\beta}^{s} \Delta_{\beta}\right) \varphi(x)_{m}=\left(-W^{1}(x)_{m}\right)^{s} \exp \left(-\beta W^{1}(x)_{m}\right) \varphi(x)_{m}, \\
& \left|\exp \left(h R^{\prime}\left|W^{1}(x)_{m}\right|\right)\left(D_{\beta}^{s} \Delta_{\beta}\right) \varphi(x)_{m}\right| \leqq \exp \left((h+1) R^{\prime}\left|W^{1}(x)_{m}\right|\right)\left|W^{1}(x)_{m}\right|^{s} \\
& \cdot \exp \left(\left(R-R^{\prime}\right)\left|W^{1}(x)_{m}\right|\right)\left|\varphi(x)_{m}\right| \\
& \leqq s !\|\varphi\|_{\delta, h+1}\left(A_{3}\right)^{s} \text {, }
\end{aligned}
$$

and the proposition is proved.

Lemma 3.4. Let $R>0$ be fixed, $\operatorname{Re} \beta^{(1+p)^{-1}}>0,|\beta|<R, \tilde{\Gamma}_{\beta}$ and $\Delta_{\beta}$ as in Propositions 3.1 and 3.3. Then the product $\widetilde{\mathbb{K}}_{\beta}=\Delta_{\beta} \tilde{\Gamma}_{\beta}$ is a bounded operator from $F_{q d, h+1}$ to $F_{(q-1) d, h}$ such that:

$$
\left\|D_{\beta}^{r} \widetilde{\mathbb{K}}_{\beta}\right\|_{q d, h+1}^{(q-1) d, h} \leqq A_{0} A^{r}((p+1) r) !
$$

uniformly for $q>1$ and $h, r \in \mathbb{N}_{0}$.

Proof. By definition of the weighted norms (27) in $F_{\delta, h}\left(\delta>0, h \in \mathbb{N}_{0}\right)$ it obviously follows from Proposition 3.2 that

$$
\left\|\left(D_{\beta}^{s} \tilde{\Gamma}_{\beta}\right) \varphi\right\|_{(q-1) d, h} \leqq A_{1}\left(A_{2}\right)^{s}((p+1) s) !\|\varphi\|_{q d, h}
$$

for all $\varphi \in F_{q d, h}$. Thus by (30) and Proposition 3.3

$$
\begin{aligned}
\left\|D_{\beta}^{r} \Delta_{\beta} \tilde{\Gamma}_{\beta}\right\|_{q d, h+1}^{(q-1) d, h} & \leqq \sum_{s=0}^{r}\left(\begin{array}{l}
r \\
s
\end{array}\right)\left\|D_{\beta}^{s} \Delta_{\beta}\right\|_{(q-1) d, h+1}^{(q-1) d, h}\left\|D_{\beta}^{r-s} \tilde{\Gamma}_{\beta}\right\|_{q d, h+1}^{(q-1) d, h+1} \\
& \leqq \sum_{s=0}^{r} 2^{r}\left(A_{3}\right)^{s} s ! A_{1}\left(A_{2}\right)^{r-s}((p+1)(r-s)) ! \\
& \leqq A_{0} A^{r}((p+1) r) !
\end{aligned}
$$

and the lemma is proved.

Lemma 3.5. For any $R>0$ there is $R_{1}>0$ such that, for $|z|<R_{1}, \varrho(\beta, z)$ admits an analytic continuation $\tilde{\varrho}(\beta, z)$ in $F_{d}$ to the region $\operatorname{Re} \beta^{(1+p)^{-1}}>0,|\beta|<R$. Moreover

$$
\left\|D_{\beta}^{r} \tilde{\varrho}(\beta, z)\right\|_{d} \leqq|z| B_{0} B^{r}((p+2) r) !
$$

uniformly with respect to $\beta, z$.

Proof. Since $\alpha\left(x_{1}\right)=1, \alpha(x)_{m}=0$ for $m>1,\|\alpha\|_{\delta, h}=1$ for all $\delta$ and $h$. Hence the partial sums of the geometric series associated with (11) satisfy:

$$
\begin{aligned}
& \left\|\sum_{h=0}^{N}\left(z \widetilde{\mathbb{K}}_{\beta}\right)^{h} z \alpha\right\|_{d} \\
& \quad \leqq \sum_{h=0}^{N}|z|^{h+1}\left\|\widetilde{\mathbb{K}}_{\beta}\right\|_{2 d, 1}^{d, 0}\left\|\widetilde{\mathbb{K}}_{\beta}\right\|_{3 d, 2}^{2 d, 1} \ldots\left\|\widetilde{\mathbb{K}}_{\beta}\right\|_{(h+1) d, h}^{h d, h-1}\|\alpha\|_{(h+1) d, h} \\
& \quad \leqq|z|\left(1-|z| A_{0}\right)^{-1}
\end{aligned}
$$

uniformly with respect to $N$, by Lemma 3.4. Thus, given $R>0$, there is $R_{1}=\left(A_{0}\right)^{-1}$ such that $\tilde{\varrho}(\beta, z)$ exists for $|\beta|<R, \operatorname{Re} \beta^{(1+p)^{-1}}>0,|z|<R_{1}$ as a uniform limit, in $F_{d}$, of analytic approximants. 
Moreover, by Lemma 3.4:

$$
\begin{aligned}
& \left\|D_{\beta}^{r} \tilde{\varrho}(\beta, z)\right\|_{d}=\left\|\sum_{h=0}^{\infty} z^{h+1} \sum_{\substack{r_{1}, \ldots, r_{n} \geq 0 \\
r_{1}+\ldots+r_{h}=r}} \frac{r !}{r_{1} ! \ldots r_{h} !}\left(D_{\beta}^{r_{1}} \widetilde{\mathbb{K}}_{\beta}\right)\left(D_{\beta}^{r_{2}} \widetilde{\mathbb{K}}_{\beta}\right) \ldots\left(D_{\beta}^{r_{h}} \widetilde{\mathbb{K}}_{\beta}\right) \alpha\right\|_{d} \\
& \leqq \sum_{h=0}^{\infty}|z|^{h+1} \sum_{\substack{r_{1}, \ldots, r_{h} \geq 0 \\
r_{1}+\ldots+r_{h}=r}} \frac{r !}{r_{1} ! \ldots r_{h} !}\left\|D_{\beta}^{r_{1}} \widetilde{\mathbb{K}}_{\beta}\right\|_{2 d, 1}^{d, 0}\left\|D_{\beta}^{r_{3}} \widetilde{\mathbb{K}}_{\beta}\right\|_{3 d, 2}^{2 d, 1} \ldots\left\|D^{r_{h}} \widetilde{\mathbb{K}}_{\beta}\right\|_{(h+1) d, h}^{h d, h-1} \\
& \leqq \sum_{h=0}^{\infty}|z|^{h+1} \sum_{\substack{r_{1}, \ldots, r_{h} \geq 0 \\
r_{1}+\ldots+r_{h}=r}} r !\left(A_{0}\right)^{h} A^{r_{1}}\left((p+1) r_{1}\right) ! \ldots A^{r_{h}}\left((p+1) r_{h}\right) ! \\
& \leqq|z|\left(B_{1}\right)^{r}\left(1-|z| A_{0}\right)^{-1} A^{r}((p+2) r) !
\end{aligned}
$$

and (32) is proved.

A bound of the type (32) can be easily extended to the function $\beta p(\beta, z)$, where $p(\beta, z)$ is the thermodynamic limit of the pressure, as well as to $f(\tilde{\varrho}(\beta, z))$, where $f$ is any linear functional defined on $F_{d}$ (see [7]). As a consequence, the remainders of the Taylor expansions of such functions satisfy the criterion for Borel-Le Roy summability of order $p+1$, which is implicit in Watson-Nevanlinna theorem concerning Borel summability (see $[2,3,6]$ ).

Theorem 3.6. If $\Phi$ satisfies assumptions (1), (2), (3), the power series expansion at $\beta=0$ of $\beta p(\beta, z)$ and $f(\tilde{\varrho}(\beta, z))$ (where $f$ is any linear functional on $F_{d}$ ) admits a convergent Borel-Le Roy sum of order $p+1$.

Acknowledgements. It is a pleasure to thank Professors V. Grecchi and G. Gallavotti for useful discussions.

\section{References}

1. Fisher, M.E., Ruelle, D.: The stability of many-particle systems. J. Math. Phys. 7, 260 (1966)

2. Graffi, S., Grecchi, V., Simon, B.: Borel summability: application to the anharmonic oscillator. Phys. Lett. 32B, 631 (1970)

3. Hardy, G.H.: Divergent series. Oxford: Clarendon Press 1949

4. Reed, M., Simon, B.: Methods of modern mathematical physics, Vol. IV. New York: Academic Press 1978

5. Ruelle, D.: Statistical mechanics. Rigorous results. New York: Benjamin 1969

6. Sokal, A.: An improvement of Watson's theorem on Borel summability. J. Math. Phys. 21, 261 (1980)

7. Wagner, W.: Borel-summability of the high temperature expansion for classical continuous systems. Commun. Math. Phys. 82, 183 (1981)

8. Wentzel, G.: Quantum theory of fields. New York: Interscience 1949

Communicated by J. Fröhlich

Received July 20, 1984 
